\title{
Radiological Stability after Revision of Infected Total Knee Arthroplasty Using Modular Metal Augments
}

\author{
Kyung-Jae Lee, MD, Ki-Cheor Bae, MD, Chul-Hyun Cho, MD, Eun-Seok Son, MD, and Jae-Won Jung, MD
}

Department of Orthopedic Surgery, Keimyung University School of Medicine, Daegu, Korea

Purpose: To evaluate the radiological stability according to the number of modular augments after revision of infected total knee arthroplasty (TKA). Materials and Methods: Between February 2006 and September 2013, 37 patients (39 knees) followed $\geq 2$ years after revision of infected TKA using modular metal augments for bone defects were reviewed retrospectively. We divided the patients into 3 groups according to the number of augments into group A ( $\leq 2$ augments, 14 knees), group B ( $3-4$ augments, 18 knees), and group C ( $5 \geq$ augments, 7 knees) and evaluated the width of radiolucent zones around the implant at the last follow-up.

Results: There were 3 Anderson Orthopedic Research Institute type I, 33 type II, and 3 type III bone defects. The mean number of radiolucent zones of group A was 3 and the sum of width averaged $4.4 \mathrm{~mm}$. In group B, the values were 4.8 and $6.2 \mathrm{~mm}$, respectively. In group C, the values were 8.1 and $12.9 \mathrm{~mm}$, respectively. The differences between the three groups were statistically significant.

Conclusions: In revision TKA with modular metal augmentation caused by infected TKA, increased modularity can result in radiological instability.

Keywords: Knee, Revision arthroplasty, Modular metal augmentation, Radiologic stability

\section{Introduction}

Bone loss is commonly encountered in revision total knee arthroplasty (TKA). Clinical success and survivorship of revision TKA are critically dependent on the restoration of bone mass and joint stability ${ }^{1)}$. Bone defects can be managed surgically, depending on the extent and location, by bone cementing, morselized bone grafting, modular metal augmentation, structural bone allografts, and custom-made implants ${ }^{2-4)}$.

Structural allografts have been associated with increased risk of fracture, nonunion, and transmission of infection and require meticulous preparation for maximizing contact with the

Received March 23, 2015; Revised (1st) August 12, 2015;

(2nd) October 13, 2015; Accepted November 3, 2015

Correspondence to: Ki-Cheor Bae, MD

Department of Orthopedic Surgery, Keimyung University School of

Medicine, 56 Dalseong-ro, Jung-gu, Daegu 41931, Korea

Tel: +82-53-250-7038, Fax: +82-53-250-7205

E-mail: bkc@dsmc.or.kr

This is an Open Access article distributed under the terms of the Creative Commons Attribution Non-Commercial License (http://creativecommons.org/licenses/by-nc/4.0/) which permits unrestricted non-commercial use, distribution, and reproduction in any medium, provided the original work is properly cited. bone graft $^{5-8)}$. On the other hand, modular metal augments are easy and quick to use through minimal bone resection and biomechanically designed to allow for joint mobility and weightbearing immediately after surgery, and thus are frequently employed in revision $\mathrm{TKA}^{9,10)}$. Metal stems are also commonly used in revision TKA for correct positioning of the knee implant, proper stress distribution of bone-implant contact surface, and rigid fixation ${ }^{11-13)}$. However, increased modularity by the use of modular metal augments may cause osteolysis of adjacent bones due to corrosion and wear at modular interfaces of the knee ${ }^{14-16)}$, eventually compromising postoperative radiological stability.

The purpose of our study was to evaluate the relationship between radiological stability and the level of modularity determined by the number of modular augments used in revision surgery for infected TKA.

\section{Materials and Methods}

\section{Patients}

A total of 37 patients (39 knees) were followed after revision surgery for infected TKA using modular metal augments for bone defects between February 2006 and September 2013. The indication for revision was infection after primary TKA in all 
knees. The revision TKA was performed in a two-stage approach: debridement and cement spacer insertion were followed by the stage two for reimplantation. There were 7 males and 30 females. Of these, one male patient and one female patient underwent revision TKA in both knees, albeit not simultaneously. The mean age of the patients was 65.8 years (range, 51 to 83 years) and the mean follow-up period was 40 months (range, 25 to 88 months). For comparison, the patients were classified into three groups according to the number of metal augments used for femoral/tibial bone defects except for the stem: group A ( $\leq 2,14$ knees), group B

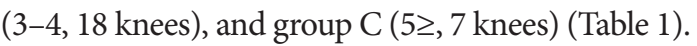

Table 1. Modular Metal Augments Distribution

\begin{tabular}{|c|c|c|c|c|}
\hline \multirow{2}{*}{ Group } & \multirow{2}{*}{ Case } & \multicolumn{2}{|c|}{ Femur (mm) } & \multirow{2}{*}{ Tibia $(\mathrm{mm})$} \\
\hline & & Posterior & Distal & \\
\hline \multirow[t]{8}{*}{$\mathrm{A}$} & 1 & & & \\
\hline & 1 & Med 4 & Med 4 & \\
\hline & 4 & & Both 4 & \\
\hline & 1 & & Both 8 & \\
\hline & 2 & & Both 5 & \\
\hline & 2 & Med 4 & & \\
\hline & 2 & Both 4 & & \\
\hline & 1 & & & Both 5 \\
\hline \multirow[t]{14}{*}{ B } & 2 & Both 4 & Both 4 & \\
\hline & 2 & Both 4 & Both 8 & \\
\hline & 2 & Both 8 & Med 8/Lat 4 & \\
\hline & 1 & Both 8 & Both 4 & \\
\hline & 1 & Both 4 & Med 4/Lat 8 & \\
\hline & 1 & Med 4 & Med 4/Lat 8 & \\
\hline & 1 & Med 8/Lat 4 & & \\
\hline & 1 & Med 4/Lat 8 & Both 8 & \\
\hline & 1 & Med 4 & & Both 10 \\
\hline & 2 & Both 8 & Both 8 & \\
\hline & 1 & Both 5 & Both 10 & \\
\hline & 1 & & Both 4 & Med 10 \\
\hline & 1 & Med 4 & Both 4 & \\
\hline & 1 & Med 4 & Med 4 & Med $8(w)$ \\
\hline \multirow[t]{6}{*}{$\mathrm{C}$} & 1 & Both 8 & Both 8 & Both 10 \\
\hline & 1 & Both 8 & Both 8 & Med 15 \\
\hline & 1 & Both 8 & Both 4 & Both 10 \\
\hline & 2 & Both 4 & Both 8 & Both 10 \\
\hline & 1 & Both 10 & Both 10 & Both 15 \\
\hline & 1 & Med 5/Lat 10 & Both 5 & Med 5/Lat 10 \\
\hline
\end{tabular}

Med: medial, Lat: lateral, w: wedge.

\section{Surgical Technique}

Skin incision was performed along the incision line for cement spacer insertion and the knee joint was exposed by medial parapatellar approach. After peri-implant soft tissue removal, the bone cement between the femoral component and the femur was separated with an osteotome and a hammer and removed using an impactor. The tibial polyethylene insert was separated from the bone cement using a microsaw and removed by cracking with an osteotome. Soft tissues around the exposed tibial and femoral cancellous bones were removed. Then, 1-2 mm resection of the protruding bone and femoral intercondylar notch was performed for placement of a posterior cruciate ligament-substituting femoral component. Swollen synovial membrane and soft tissues were debrided and thoroughly lavaged. The surgery was a two-stage procedure in all knees and bone allograft was ruled out as an option for the management of bone loss. For restoration of bone mass, bone cement was used in the knees with Anderson Orthopedic Research Institute (AORI) type I bone defects, modular metal augments were used in addition to bone cement in the knees with AORI type II or III bone defects. The knee components, metal augments, and stems were all fixed with metal screws. Of the total 42 knees, the prosthesis of choice was Press Fit Condylar Sigma Total Condylar 3 (Johnson \& Johnson, Warsaw, IN, USA) in 37 knees, Scorpio Total Stabilizer (Stryker, Mahway, NJ, USA) in 3 knees, and NexGen Legacy Constrained Condylar Knee (Zimmer, Warsaw, IN, USA) in 2 knees. For stable component fixation, the femoral/tibial stems were fully cemented with Depuy CMW Gentamicin 3 bone cement (Depuy, Blackpool, UK) containing $4.22 \% \mathrm{w} / \mathrm{w}$ of gentamicin sulfate in all knees. The patella was resurfaced in all knees.

\section{Radiological Assessment}

Radiological stability was evaluated using a modified roentgenographic method of the American Knee Society according to Whaley et al. ${ }^{17}$ : radiolucent zones and bone resorption around the implants of the femur and tibia excluding the patella were investigated on the anteroposterior and lateral radiographs of the knee $^{17)}$. In addition, the distribution and extent of radiolucency and bone resorption were assessed according to the number of metal augments.

\section{Statistical Analysis}

Statistical analysis was performed using SPSS ver. 18.0 (SPSS Inc., Chicago, IL, USA). Analysis of variance analysis was used for comparison of radiological stability after revision TKA according to the number of metal augments. The relationship 
between the use of metal augments and radiological stability was evaluated using Mann-Whitney test. Statistical significance was set at $\mathrm{p}<0.05$.

\section{Results}

\section{AORI Classification}

According to the AORI classification, there were 3 F1T1 knees, 1 F1T2A knee, 2 F1T2B knees, 3 F2AT1 knees, 21 F2BT1 knees, 1 F2AT2A knee, 1 F2BT2A knee, 4 F2BT2B knees, and 3 F2BT3 knees (Table 2).

\section{Radiological Outcome}

At the last follow-up, radiolucency was present in 34 of 39 knees (87.1\%) and component loosening was not observed in all knees. The mean number of radiolucent zones was 4.7 (range, 0 to 15) and the average sum of width was $6.7 \mathrm{~mm}$ (range, 0 to $23 \mathrm{~mm}$ ): the mean number and width were 3 (range, 0 to 8 ) and $4.4 \mathrm{~mm}$ (range, 0 to $13 \mathrm{~mm}$ ) in group A; 4.8 (range, 0 to 14 ) and $6.2 \mathrm{~mm}$ (range, 0 to $18 \mathrm{~mm}$ ) in group $B$; and 8.1 (range, 1 to 15 ) and 12.9 $\mathrm{mm}$ (range, 1 to $23 \mathrm{~mm}$ ) in group C. The number of metal augments used in revision TKA was positively correlated with the number of radiolucent zones and the sum of width (Fig. 1).

Compared to the knees where metal augmentation was applied only to the femur, those with metal augments around the tibia and femur showed statistically significant increases in the number of radiolucent zones and width around the tibia; however, there was no notable difference between the knees with respect to radiolucency around the femur (Table 3).

In a 68-year-old female patient who presented with delayed chronic infection of the left knee, 4-mm blocks were used for bone defects in the medial and lateral areas of the distal femur during two-stage revision TKA. At 25 months after revision, no radiolucent line was observed in her knee (Fig. 2). In a 69-yearold female patient who underwent the revision surgery due to delayed chronic infection following TKA, four bone blocks were used for bone deficiency in the femur during revision TKA:

Table 2. Bone Defects of Patients

\begin{tabular}{cccc}
\hline & \multicolumn{3}{c}{ Tibia } \\
\cline { 2 - 4 } & AORI 1 & AORI 2 & AORI 3 \\
\hline Femur & 3 & 3 & 0 \\
AORI 1 & 24 & 6 & 3 \\
AORI 2 & & & \\
\hline
\end{tabular}

AORI: Anderson Orthopaedic Research Institute. 8-mm blocks in the medial and lateral areas of the posterior aspect of the femur, an 8-mm block in the medial area of the distal femur, and a 4-mm block in the lateral area of the distal femur. At 26 months after surgery, 7-mm radiolucency was observed in 5 zones in the patient (Fig. 3). In a 71-year-old female patient, twostage revision TKA was performed for delayed chronic infection using 6 metal augments: 8-mm blocks in the medial and lateral areas of both the posterior aspect of the femur and distal femur and $10-\mathrm{mm}$ blocks in the medial and lateral areas of the tibia. In the patient, 11-mm radiolucency was observed in 7 zones at 48 months after surgery (Fig. 4).

\section{Discussion}

A number of domestic and international studies have been published on the management of bone deficiency during revision TKA and treatment outcomes. Among various methods, modular metal augmentation has demonstrated favorable results for AORI type II bone defects in several studies ${ }^{4,16,18-21)}$. According to some recent studies, metal augmentation showed promising results for restoration of bone mass in primary as well ${ }^{14,22}$. Tsukada et al. ${ }^{22}$ reported that the clinical results of primary TKA with metal augmentation in patients with bone defects were comparable to those without augmentation in patients without bone defects. Accordingly, metal augmentation has been considered as a convenient and effective method for restoration of bone mass. However, fretting at the metal augment-implant interface and component loosening have been documented as major shortcomings of metal augmentation. In particular, the appearance of radiolucent lines after metal augmentation has been reported in several studies focused on the relationship between the clinical outcome and radiological stability ${ }^{4,15,18,19,22)}$.

Pagnano et al. ${ }^{16)}$ reported that radiolucency was noted in 13 of

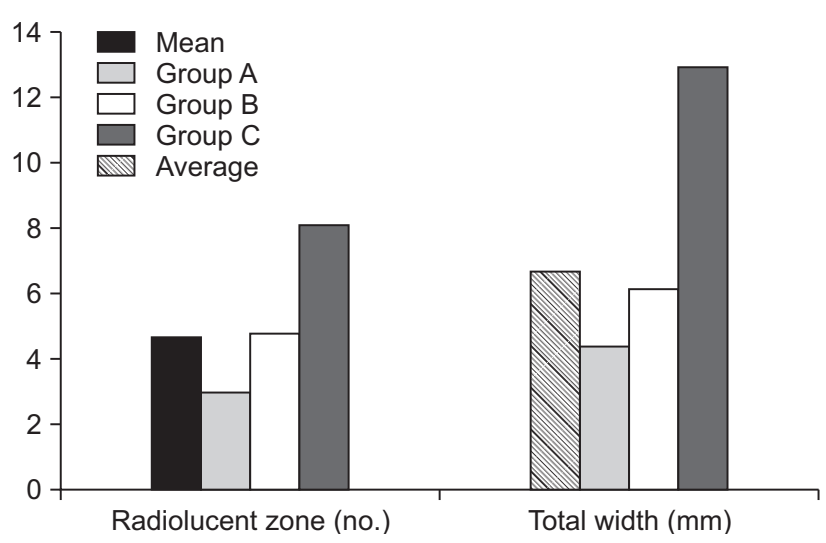

Fig. 1. Number of the radiolucent zones and sum of the widths. 
Table 3. Difference of the Radiolucent Line According to the Use of Tibia Modular Metal Augments

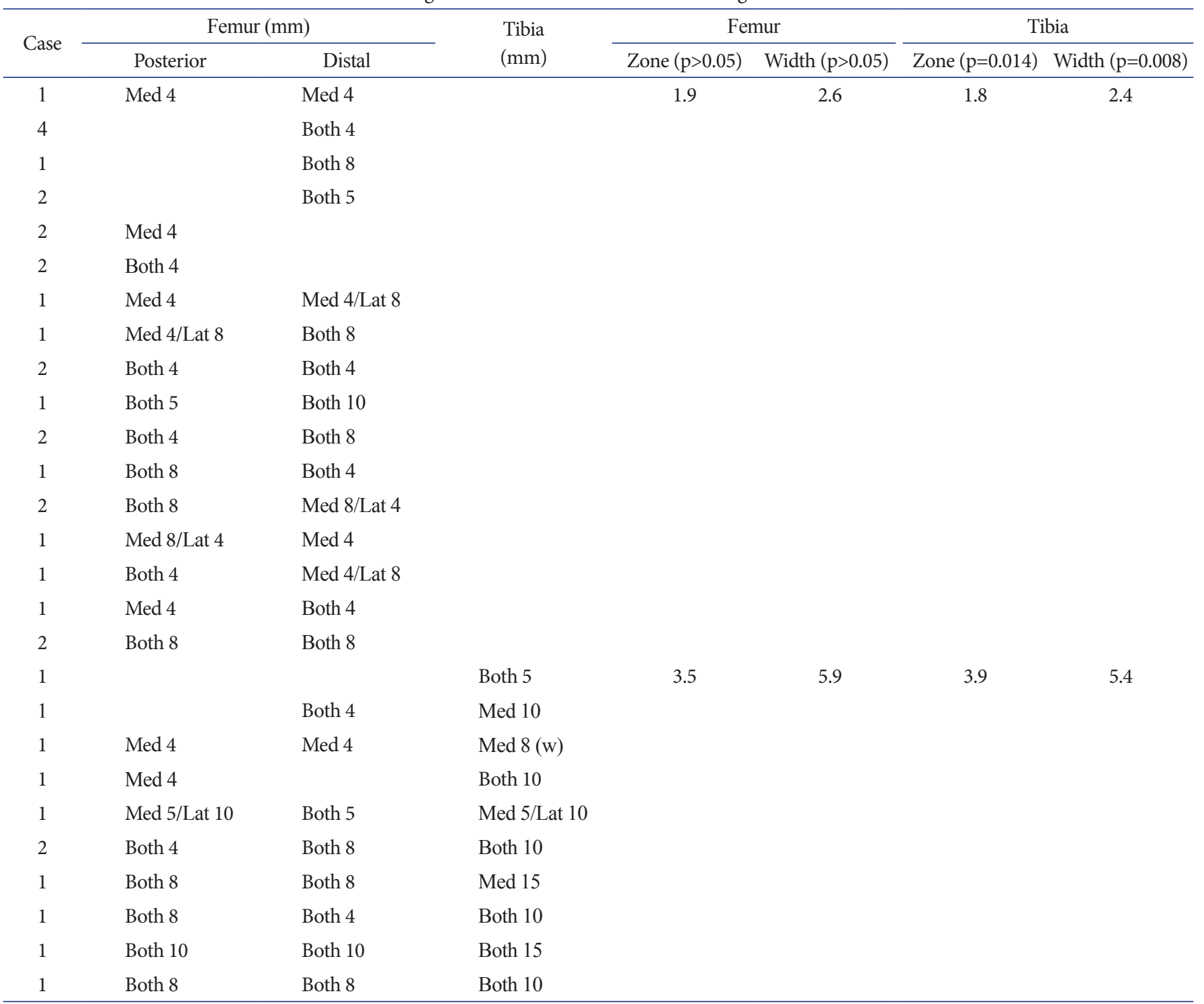

Med: medial, Lat: lateral, w: wedge.
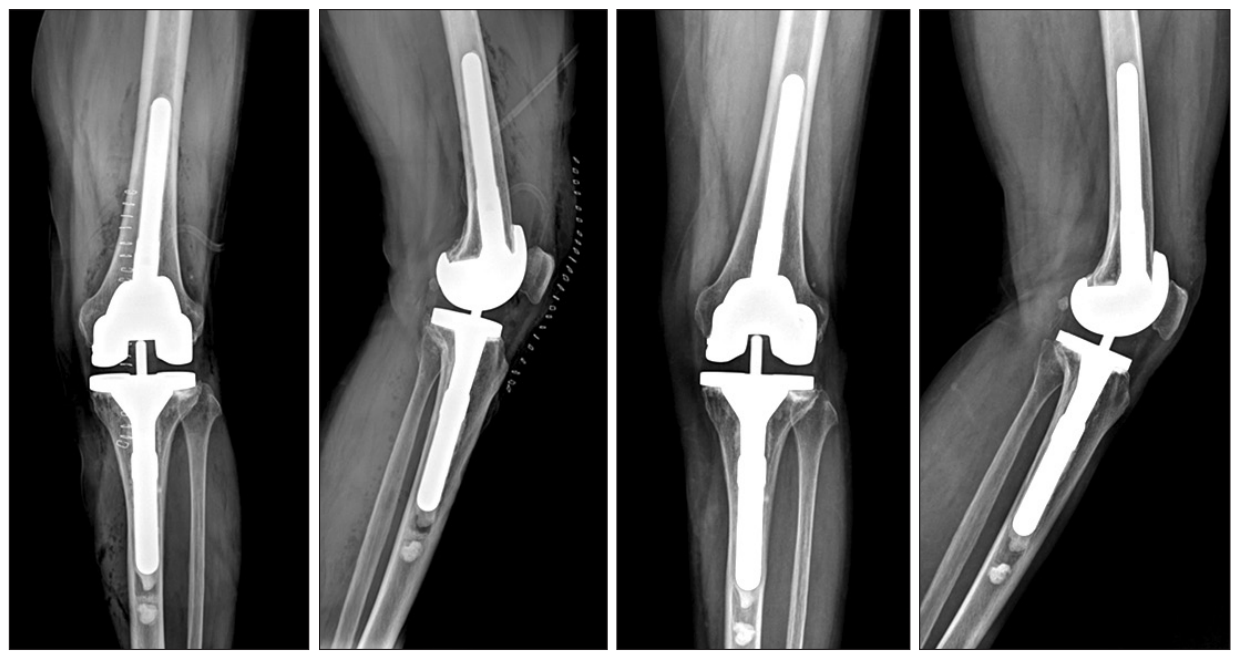

Fig. 2. A 68-year-old female, group A: 25 months after revision total knee arthroplasty, there is no radiolucent line. 

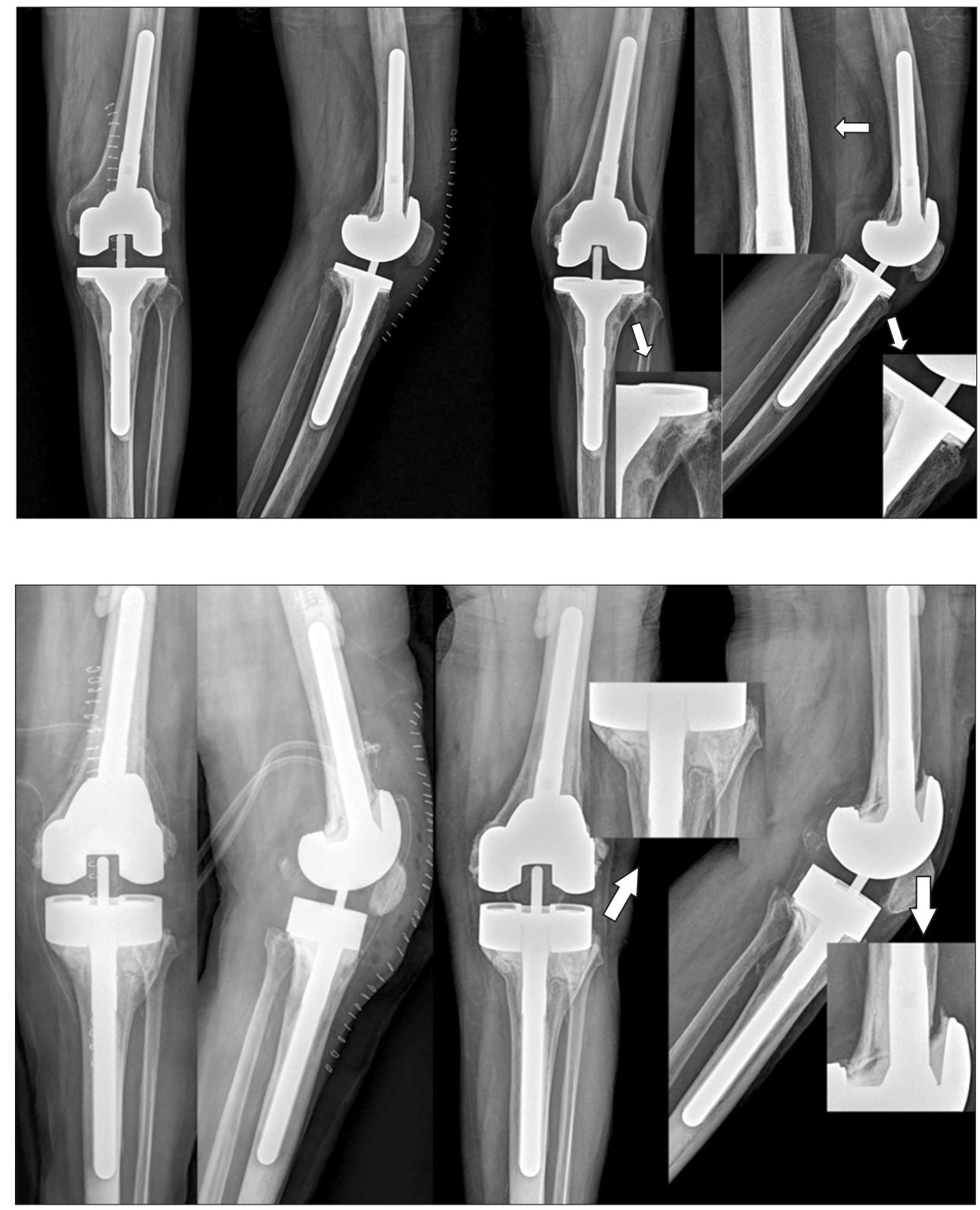

Fig. 3. A 69-year-old female, group B: 26 months after revision total knee arthroplasty, there is a $3-\mathrm{mm}$ radiolucent line in zone 3 of the tibia on the anterior to posterior radiograph, a 1-mm radiolucent line in zone 1 of the tibia, and a total 3-mm radiolucent line in zones $5 b, 6 a$, and $7 b$ of the femur on the lateral radiograph.
Fig. 4. A 71-year-old female, group C: 48 months after revision total knee arthroplasty, there is bone resorption of approximately $4 \mathrm{~mm}$ in zone 1 of the femur, a total $3 \mathrm{~mm}$ in zones 1 and 2 of the tibia on the lateral radiograph, and a total $4-\mathrm{mm}$ radiolucent line in zones 1,2,5, and 7 of the tibia on the anterior to posterior radiograph.
24 knees with metal wedge augments for tibial bone deficiency after primary TKA. Haas et al. ${ }^{23)}$ observed progressive radiolucent lines around the femoral component in $1 \%$ of the patients and around the tibial component in 3\% of the patients at a mean of 42 months after revision TKA using metal augments and cementless stemmed implants. Patel et al. ${ }^{19)}$ described that radiolucent lines were present in $14 \%$ of the 79 knees at a mean of 7 years after revision TKA using metal augments and hybrid fixation stems.

Although the etiology of radiolucency after TKA has yet to be established, possible causes include insufficient bone cementing, thermal bone necrosis during hardening of the cement, presence of blood or tissue debris, and micromotion of knee implants ${ }^{24)}$. Tsukada et al. ${ }^{22)}$ suggested that appearance of radiolucency can be related to 1) valgus alignment of the operated leg where stress shielding occurs due to decreased stress on the medial part of the knee and 2) the use of a stem that transmits the load from the tibial surface to the distal area resulting in less stress on the metal augment.

In general, metal augmentation is considered necessary for implant stability if $\geq 40 \%$ of the bone-implant interface is not supported by host bone ${ }^{4)}$. In our study, AORI type 2 or type 3 bone defects were present in all knees $(n=36)$ and the bone-implant interface was insufficient in all cases at the time of revision TKA. Accordingly, metal augmentation combined with bone cementing was performed for restoration of bone mass during revision surgery. Unfortunately, fretting may occur at modular interfaces 
and the resultant metal debris may cause the development of osteolysis of the surrounding bone and radiolucent lines ${ }^{14-16,19,25)}$. In this study, therefore, we attempted to investigate the relationship between radiological stability and metal augmentation. The results of the current study show that the larger the number of metal augments (the larger the interfaces between the metal augments and the implants), the greater the radiolucency around the implants in terms of the zone number and width. Such a finding can also be supported by the significant increase in the zone number and width of radiolucency around the tibia only, not the femur, in the knees with metal augmentation for tibial bone defects when compared to those without metal augmentation of the tibia. Although it is too early to implicate metal augmentation as the major cause of radiolucency after revision TKA, we think that it could be associated with postoperative radiolucency assuming that the greater the number of metal augments, the higher the risk of corrosion and wear of the augments. Still, we admit that no experimental data exist to validate our understanding on the etiology of radiolucency.

The incidence of radiolucency (35/36) was higher in the current study comparted to that in previous studies ${ }^{19,23)}$. In our opinion, this can be attributed to the use of full-cemented stem that is vulnerable to stress shielding effect in the distal femur and proximal tibia ${ }^{26)}$.

Panni et al. ${ }^{4)}$ reported that there was no statistically significant difference after revision TKA using metal augments with respect to aseptic loosening or polyethylene wear between the infected knees and the uninfected knees. Lee et al. ${ }^{27)}$ described there was remarkable clinical difference between infected knees and uninfected knees, but there was no notable intergroup difference in terms of radiolucency. In the current study, the indication for the two-stage revision TKA was infection in all knees, which might be attributable to the higher incidence of radiolucency compared to that in other studies; however, the influence of infection should be investigated in further research involving comparison with revision TKA patients with non-infectious causes.

The limitations of this study include the retrospective study design, short follow-up period, small sample size, and lack of comparison with revision TKA patients without infection. In addition, there were no experimental data pertaining to the relationship between the development of radiolucency and the number of metal augments. Lastly, in our opinion, comparison of long-term survivorship among patients with different numbers of metal augments should be addressed in further research.

\section{Conclusions}

Metal augmentation for management of bone deficiency in revision surgery after infected TKA has been recognized as an effective method for clinical improvement. However, our findings demonstrate a negative correlation between the number of augments and radiological stability.

\section{Conflict of Interest}

No potential conflict of interest relevant to this article was reported.

\section{References}

1. Completo A, Simoes JA, Fonseca F, Oliveira M. The influence of different tibial stem designs in load sharing and stability at the cement-bone interface in revision TKA. Knee. 2008;15:227-32.

2. Ghazavi MT, Stockley I, Yee G, Davis A, Gross AE. Reconstruction of massive bone defects with allograft in revision total knee arthroplasty. J Bone Joint Surg Am. 1997;79:17-25.

3. Gross AE. Revision total knee arthroplasty of bone grafts versus implant supplementation. Orthopedics. 1997;20:843-4.

4. Panni AS, Vasso M, Cerciello S. Modular augmentation in revision total knee arthroplasty. Knee Surg Sports Traumatol Arthrosc. 2013;21:2837-43.

5. Bauman RD, Lewallen DG, Hanssen AD. Limitations of structural allograft in revision total knee arthroplasty. Clin Orthop Relat Res. 2009;467:818-24.

6. Clatworthy MG, Ballance J, Brick GW, Chandler HP, Gross AE. The use of structural allograft for uncontained defects in revision total knee arthroplasty: a minimum five-year review. J Bone Joint Surg Am. 2001;83:404-11.

7. Dennis DA. The structural allograft composite in revision total knee arthroplasty. J Arthroplasty. 2002;17(4 Suppl 1): 90-3.

8. Whittaker JP, Dharmarajan R, Toms AD. The management of bone loss in revision total knee replacement. J Bone Joint Surg Br. 2008;90:981-7.

9. Hockman DE, Ammeen D, Engh GA. Augments and allografts in revision total knee arthroplasty: usage and outcome using one modular revision prosthesis. J Arthroplasty. 2005;20:35-41.

10. Lucey SD, Scuderi GR, Kelly MA, Insall JN. A practical approach to dealing with bone loss in revision total knee ar- 
throplasty. Orthopedics. 2000;23:1036-41.

11. Beckmann J, Luring C, Springorum R, Kock FX, Grifka J, Tingart M. Fixation of revision TKA: a review of the literature. Knee Surg Sports Traumatol Arthrosc. 2011;19:872-9.

12. Conditt MA, Parsley BS, Alexander JW, Doherty SD, Noble PC. The optimal strategy for stable tibial fixation in revision total knee arthroplasty. J Arthroplasty. 2004;19(7 Suppl 2): 113-8.

13. Mulhall KJ, Ghomrawi HM, Scully S, Callaghan JJ, Saleh KJ. Current etiologies and modes of failure in total knee arthroplasty revision. Clin Orthop Relat Res. 2006;446:45-50.

14. Baek SW, Kim CW, Choi CH. Management of tibial bony defect with metal block in primary total knee replacement arthroplasty. Knee Surg Relat Res. 2013;25:7-12.

15. Brand MG, Daley RJ, Ewald FC, Scott RD. Tibial tray augmentation with modular metal wedges for tibial bone stock deficiency. Clin Orthop Relat Res. 1989;(248):71-9.

16. Pagnano MW, Trousdale RT, Rand JA. Tibial wedge augmentation for bone deficiency in total knee arthroplasty. A followup study. Clin Orthop Relat Res. 1995;(321):151-5.

17. Whaley AL, Trousdale RT, Rand JA, Hanssen AD. Cemented long-stem revision total knee arthroplasty. J Arthroplasty. 2003;18:592-9.

18. Lee MC, Seong SC, Jo HC, Lee SH, Jang JD, Park SE, Lim ST, Hwang CJ. The management of bone defects in revision total knee arthroplasty. J Korean Orthop Assoc. 2002;37:197-203.

19. Patel JV, Masonis JL, Guerin J, Bourne RB, Rorabeck CH. The fate of augments to treat type- 2 bone defects in revision knee arthroplasty. J Bone Joint Surg Br. 2004;86:195-9.

20. Stulberg SD. Bone loss in revision total knee arthroplasty: graft options and adjuncts. J Arthroplasty. 2003;18(3 Suppl 1): 48-50.

21. Rand JA. Modular augments in revision total knee arthroplasty. Orthop Clin North Am. 1998;29:347-53.

22. Tsukada S, Wakui M, Matsueda M. Metal block augmentation for bone defects of the medial tibia during primary total knee arthroplasty. J Orthop Surg Res. 2013;8:36.

23. Haas SB, Insall JN, Montgomery W 3rd, Windsor RE. Revision total knee arthroplasty with use of modular components with stems inserted without cement. J Bone Joint Surg Am. 1995;77:1700-7.

24. Guha AR, Debnath UK, Graham NM. Radiolucent lines below the tibial component of a total knee replacement (TKR): a comparison between single-and two-stage cementation techniques. Int Orthop. 2008;32:453-7.

25. Lohmann CH, Singh G, Willert HG, Buchhorn GH. Metallic debris from metal-on-metal total hip arthroplasty regulates periprosthetic tissues. World J Orthop. 2014;5:660-6.

26. Shannon BD, Klassen JF, Rand JA, Berry DJ, Trousdale RT. Revision total knee arthroplasty with cemented components and uncemented intramedullary stems. J Arthroplasty. 2003; 18(7 Suppl 1):27-32.

27. Lee KJ, Moon JY, Song EK, Lim HA, Seon JK. Minimum two-year results of revision total knee arthroplasty following infectious or non-infectious causes. Knee Surg Relat Res. 2012;24:227-34. 